\title{
Integrability and continuity of functions represented by trigonometric series: coefficients criteria
}

by

\author{
Mikhail Dyachenko (Moscow) and Sergey Tikhonov (Barcelona)
}

\begin{abstract}
We study weighted $L_{p}$-integrability $(1 \leq p<\infty)$ of trigonometric series. It is shown how the integrability of a function with weight $x^{-\alpha}$ depends on some regularity conditions on Fourier coefficients. Criteria for the uniform convergence of trigonometric series in terms of their coefficients are also studied.
\end{abstract}

1. Introduction. Throughout this paper we shall let $f(x)$ and $g(x)$ denote the sums of the series

$$
\sum_{n=1}^{\infty} a_{n} \cos n x
$$

and

$$
\sum_{n=1}^{\infty} b_{n} \sin n x
$$

respectively, whenever they exist. The paper concerns two main questions.

Problem 1. If $f, g \in L_{1}$, what hypotheses on $\left\{a_{n}\right\},\left\{b_{n}\right\}$ are equivalent to (imply, follow from)

$$
|f(x)|^{p} x^{-\alpha} \in L \quad \text { or } \quad|g(x)|^{p} x^{-\alpha} \in L ?
$$

This question is very well studied (see $[\mathrm{Bo}],[\mathrm{St}],[\mathrm{Zy}]$ ) and many classical results on Fourier coefficients such as the Hardy-Littlewood-Paley theorem, Pitt's inequality, and the Young-Heywood-Boas results show that in many cases the appropriate condition on the coefficients is

$$
\sum_{n=1}^{\infty} n^{\alpha+p-2}\left|a_{n}\right|^{p}<\infty \quad \text { or } \quad \sum_{n=1}^{\infty} n^{\alpha+p-2}\left|b_{n}\right|^{p}<\infty .
$$

2000 Mathematics Subject Classification: Primary 42A16, 42A20; Secondary 41A17.

Key words and phrases: trigonometric series, weighted integrability, uniform convergence, generally monotone sequences, trigonometric approximation. 
For the case $p=1$ many authors used the following convenient fact on the multipliers $x^{-\alpha}$ and $n^{\alpha-1}$ : when $0<\alpha<1$, the function $x^{-\alpha}$ has a Fourier series whose coefficients behave at infinity like $n^{\alpha-1}$, and conversely. Using this, Heywood $\left[\mathrm{He}_{2}\right]$ proved that (1.4) implies (1.3). It is known that the converse holds under monotonicity of either $\left\{a_{n}\right\},\left\{b_{n}\right\}$, or $f, g$. In Section 2 we show that, generally, without the monotonicity condition, the converse does not hold even in a much weaker form.

In Section 3 we consider the case of $1<p<\infty$ and study one of the most important results in the theory of weighted Fourier inequalities, Pitt's theorem: (1.4) implies (1.3) for $\max (0,2-p) \leq \alpha<1$. Because of the great importance of this theorem, we reprove it using the multipliers $x^{-\alpha}, n^{\alpha-1}$. In a very simple proof we apply only Hardy's inequalities and do not invoke the interpolation technique (see $[\mathrm{St}]$ ).

In Section 4 we study trigonometric series with some regularity conditions on the coefficients. Our idea is to consider quantitative characteristics of the condition of bounded variation $\left(\sum\left|\Delta a_{k}\right|<\infty\right)$, i.e.,

$$
\sum_{k=n}^{\infty}\left|\Delta a_{k}\right|<C \beta_{n}
$$

and

$$
\sum_{k=n}^{2 n-1}\left|\Delta a_{k}\right|<C \beta_{n} .
$$

Here $\beta=\left\{\beta_{n}\right\}$ is a majorant, that is, a positive sequence; and $C$ is a positive number independent of $n$.

We denote by $\overline{\operatorname{GM}}(\beta)$ and $\operatorname{GM}(\beta)$ the collections of all null-sequences (generally speaking, complex) satisfying (1.5) and (1.6), respectively. Such sequences are said to be generally monotone sequences with majorant $\beta$. Note that a class similar to $\overline{\mathrm{GM}}(\beta)$ was introduced by Leindler (see $\left[\mathrm{Le}_{2}\right.$ ] $\left(\beta_{n}=a_{n}\right)$ and $\left.\left[\mathrm{Le}_{3}\right]\right)$. The class $\operatorname{GM}(\beta)$ was introduced in $\left[\mathrm{Ti}_{1}\right]$ (see also [LZ], [ $\left.\mathrm{Ti}_{2}\right]$ for certain $\beta_{n}$, and the history of the topic in $\left.\left[\mathrm{Ti}_{3}\right]\right)$.

In Section 4 we prove that for $a, b \in \overline{\mathrm{GM}}(\beta)$ with appropriate $\beta$, conditions (1.3) and (1.4) are equivalent.

Problem 2. What hypotheses on $\left\{a_{n}\right\},\left\{b_{n}\right\}$ are equivalent to (imply, follow from) the uniform convergence of series (1.1) and (1.2)?

For the cosine series with non-negative coefficients, the answer is clearly $\sum a_{n}<\infty$. For the sine series, the classical result of Chaundy and Joliffe (see [CJ], [Zy, Vol. 1, p. 183]) states that if $\left\{b_{n}\right\}$ is monotonic, then (1.2) converges uniformly if and only if $\lim _{n \rightarrow \infty} n b_{n}=0$. In Section 5 we prove a similar result for a fairly wider class of sequences $\left\{b_{n}\right\}$, precisely for generally 
monotone sequences from the class

$$
\left\{b_{n}: \sum_{k=n}^{2 n-1}\left|\Delta b_{k}\right| \leq \frac{C}{n} \max _{k \geq[n / c]} \sum_{s=k}^{2 k} b_{s} \text { for some } c>1\right\} .
$$

This extends many known results (see, e.g., [DT $]$, [ $\left.\mathrm{Ti}_{3}\right]$, [YZZ]). In Section 6, we apply this criterion to the following approximation theory problem: find interrelations between the moduli of smoothness $\omega_{k}(\psi, 1 / n)$ and the best approximation $E_{n}(\psi)$ in $C$. It will be shown that the Jackson inequality

$$
E_{n}(\psi) \leq C \omega_{k}(\psi, 1 / n)
$$

and the weak-type inequality

$$
\omega_{k}(\psi, 1 / n) \leq \frac{C}{n^{k}} \sum_{\nu=0}^{n-1}(\nu+1)^{k-1} E_{\nu}(\psi)
$$

(see, e.g., [DL, Ch. 7]) can be sharpened as follows: for even/odd functions represented by trigonometric series with generally monotone coefficients and for even/odd $k$,

$$
\omega_{k}(\psi, 1 / n) \asymp n^{-k} \max _{\nu \in[0, n]}(\nu+1)^{k} E_{\nu}(\psi)
$$

and

$$
\omega_{k}(\psi, 1 / n) \asymp n^{-k} \sum_{\nu=0}^{n-1}(\nu+1)^{k-1} E_{\nu}(\psi) .
$$

We conclude with Section 6, where we provide a few remarks. In particular, we prove that our findings concerning the general monotonicity concept (in Sections 4 and 5) do indeed generalize all known results.

Throughout this paper, we denote by $C, C_{i}, c$ positive constants that may be different on different occasions. In addition, $F \asymp G$ means that $F \leq C G$ and $G \leq C F$.

2. Weighted $L_{1}$-integrability. We start with well-known results on interrelation between the $L_{1}$-integrability of $f(x) x^{-\alpha}$ and $g(x) x^{-\alpha}$ and the summability properties of the coefficients $\left\{a_{n}\right\}$ and $\left\{b_{n}\right\}$. The best reference for Theorems 2.1-2.3 below is the monograph by Boas [Bo].

TheOREM 2.1 ([He 2$])$. Let $\alpha \in(0,1]$. Let also $f(x) x^{-\alpha} \in L(0, \pi)$. Then for $a_{n}=a_{n}(f)$ the series $\sum_{n=1}^{\infty} a_{n} n^{\alpha-1}$ converges. If $\alpha \in(-1,1]$, then $a$ similar result holds true for the sine series as well.

To prove the converse, we have to assume some conditions on the function or on the sequence of the Fourier coefficients.

THEOREM 2.2. Let $\alpha \in(0,1)$. Assume that one of the following two conditions holds true: 
(1) the function $f \in L$ is non-negative on $(0, \delta)$ for some $\delta>0$;

(2) the sequence $\left\{a_{n}(f)\right\}$ is non-increasing.

Then the convergence of the series $\sum_{n=1}^{\infty} a_{n}(f) n^{\alpha-1}$ implies that $f(x) x^{-\alpha} \in$ $L(0, \pi)$. A similar result with $\alpha \in[0,1)$ holds true for the sine series as well.

Analogous results can be given for absolute convergence, that is,

$$
\sum_{n=1}^{\infty}\left|a_{n}(f)\right| n^{\alpha-1}<\infty .
$$

Theorem 2.3. Let $\alpha \in(0,1)$. Assume that the function $f \in L$ is nonincreasing on $(0, \delta)$ and $f(x) \geq C$ on $(\delta, \pi)$. Then the condition $f(x) x^{-\alpha} \in$ $L(0, \pi)$ is equivalent to (2.1). A similar result with $\alpha \in[0,1)$ holds true for the sine series as well.

Below we give an example which shows that both statements in Theorems 2.2 and 2.3 can fail rather dramatically when we do not assume the given conditions on $f / g$ or $\left\{a_{n}\right\} /\left\{b_{n}\right\}$. This answers some questions by Boas [Bo].

THEOREM 2.4. There exists a function $\psi(x) \in L(0,2 \pi)$ such that $\psi(x)$ is uniformly bounded on any interval $[\varepsilon, 2 \pi], \varepsilon>0$, and $x^{-\beta} \psi(x) \notin L(0,2 \pi)$ for $\beta>0$ but

$$
\sum_{n=1}^{\infty} n^{-\alpha}\left(\left|a_{n}(\psi)\right|+\left|b_{n}(\psi)\right|\right)<\infty \quad \text { for any } \alpha>0 .
$$

Proof. Let us consider the function sequence

$$
\psi_{k}(x)=\frac{k}{\ln ^{2} k} \cos \left(2^{k} x\right) \chi_{(1 /(k+1), 1 / k]}(x), \quad k \geq 3,
$$

and define for $x \in(0,2 \pi]$ a $2 \pi$-periodic function $\psi(x)$ by

$$
\psi(x)=\sum_{k=3}^{\infty} \psi_{k}(x) .
$$

Then $\psi(x) \in L(0,2 \pi)$ and $\psi(x)$ is uniformly bounded on any interval $[\varepsilon, 2 \pi]$, where $\varepsilon>0$. Moreover, $x^{-\beta} \psi(x) \notin L(0,2 \pi)$ for any positive $\beta$.

Now we estimate the sum

$$
J(\alpha) \equiv \sum_{n=1}^{\infty} n^{-\alpha}\left|a_{n}(\psi)\right|,
$$

where $\alpha$ is a positive number. Note that

$$
J(\alpha) \leq \sum_{n=1}^{\infty} n^{-\alpha} \sum_{k=3}^{\infty}\left|a_{n}\left(\psi_{k}\right)\right|=\sum_{k=3}^{\infty} \sum_{n=1}^{\infty} n^{-\alpha}\left|a_{n}\left(\psi_{k}\right)\right| \equiv \sum_{k=3}^{\infty} J_{k}(\alpha) .
$$


Let us estimate $\left|a_{n}\left(\psi_{k}\right)\right|$. For $k \geq 3$ and $\left|n-2^{k}\right| \geq k$, we get

$(2.2) \quad\left|a_{n}\left(\psi_{k}\right)\right|$

$$
\begin{aligned}
& \leq \frac{C k}{\ln ^{2} k}\left(\left|\int_{1 /(k+1)}^{1 / k} \cos \left(2^{k}-n\right) x d x\right|+\left|\int_{1 /(k+1)}^{1 / k} \cos \left(2^{k}+n\right) x d x\right|\right) \\
& \leq \frac{C k}{\left|2^{k}-n\right|} .
\end{aligned}
$$

In the case of $k \geq 3$ and $\left|n-2^{k}\right|<k$, we obtain

$$
\left|a_{n}\left(\psi_{k}\right)\right| \leq \frac{C k}{\ln ^{2} k}\left(\frac{1}{k}-\frac{1}{k+1}\right) \leq \frac{C}{k} .
$$

Therefore, for any $\alpha>0$ and $k \geq 3$, by (2.2) and (2.3), we estimate

$$
\begin{aligned}
J_{k}(\alpha) & \leq C\left(k \sum_{n=1}^{2^{k}-k} \frac{n^{-\alpha}}{2^{k}-n}+\sum_{n=2^{k}-k+1}^{2^{k}+k-1} \frac{n^{-\alpha}}{k}+k \sum_{n=2^{k}+k}^{\infty} \frac{n^{-\alpha}}{n-2^{k}}\right) \\
& \equiv C\left(J_{k, 1}+J_{k, 2}+J_{k, 3}\right) .
\end{aligned}
$$

First,

$$
J_{k, 1} \leq 2^{-k+1} k \sum_{n=1}^{2^{k-1}} n^{-\alpha}+2^{-\alpha(k-1)} k \sum_{n=2^{k-1}+1}^{2^{k}-k} \frac{1}{2^{k}-n} \leq C(\alpha) k^{2} 2^{-\alpha k}
$$

Second,

$$
J_{k, 2} \leq C(\alpha) k 2^{-\alpha(k-1)} \frac{1}{k}=C(\alpha) 2^{-\alpha k} .
$$

Finally,

$$
J_{k, 3} \leq 2^{-\alpha k} k \sum_{n=2^{k}+k}^{2^{k+1}} \frac{1}{n-2^{k}}+2 k \sum_{n=2^{k+1}+1}^{\infty} n^{-\alpha-1} \leq C(\alpha) k^{2} 2^{-\alpha k} .
$$

Then inequalities (2.4)-(2.7) imply

$$
J_{k}(\alpha) \leq C(\alpha) k^{2} 2^{-\alpha k},
$$

and hence $J(\alpha)<\infty$. Similarly we estimate the series with the sine coefficients $\sum_{n=1}^{\infty} n^{-\alpha}\left|b_{n}(\psi)\right|$. The proof is now complete.

3. Weighted $L_{p}$-integrability: Pitt's theorem. In this section we give a simple proof of Pitt's theorem [Pi]. Note that we make no use of interpolation technique (see, e.g., [St]). 
Theorem 3.1. Let $1<p<\infty$ and $\max (0,2-p) \leq \alpha<1$. Suppose $\psi(x) \in L(0, \pi)$ and

$$
\sum_{n=1}^{\infty} n^{\alpha+p-2}\left(\left|a_{n}(\psi)\right|^{p}+\left|b_{n}(\psi)\right|^{p}\right)<\infty .
$$

Then $|\psi(x)|^{p} x^{-\alpha} \in L(0,2 \pi)$.

Proof. For simplicity let $b_{n}=0$ and $\psi=f$. We assume first that $p \in$ $[2, \infty)$. Then $\alpha>0$ and we define $\varphi(x)=x^{-\alpha / p}$. By the classical Paley theorem [Zy, Ch. XII, §3],

$$
\sum_{n}\left|a_{n}(f)\right|^{p} n^{p-2}<\infty \Rightarrow f(x) \in L_{p} .
$$

Noting that $x^{-\frac{\alpha}{p} \frac{p}{p-1}} \in L(0, \pi)$ for $0<\alpha<p-1$, we get $h(x)=f(x) x^{-\alpha / p} \in$ $L(0, \pi)$. The cosine coefficients of $h(x)$ are computed from

$$
\begin{aligned}
a_{n}(h)=\frac{a_{n}(\varphi) a_{0}(f)}{2} & +\sum_{r=1}^{n} \frac{a_{n-r}(\varphi)+a_{n+r}(\varphi)}{2} a_{r}(f) \\
& +\sum_{r=n+1}^{\infty} \frac{a_{r-n}(\varphi)+a_{r+n}(\varphi)}{2} a_{r}(f) .
\end{aligned}
$$

It is known [Zy, Vol. $1, \mathrm{~V}, 2]$ that $a_{r}(\varphi) \sim r^{\alpha / p-1}$ as $r \rightarrow \infty$. Hence (see $(3.2))$,

$$
\begin{aligned}
\left|a_{n}(h)\right| \leq & C\left(\sum_{r=0}^{n}(n-r+1)^{\alpha / p-1}\left|a_{r}(f)\right|+\sum_{r=n+1}^{\infty}(r-n)^{\alpha / p-1}\left|a_{r}(f)\right|\right) \\
\leq & C_{1}\left(n^{\alpha / p-1} \sum_{r=0}^{[n / 2]}\left|a_{r}(f)\right|+\sum_{r=[n / 2]+1}^{n}(n-r+1)^{\alpha / p-1}\left|a_{r}(f)\right|\right. \\
& \left.+\sum_{r=n+1}^{2 n}(r-n)^{\alpha / p-1}\left|a_{r}(f)\right|+\sum_{r=2 n+1}^{\infty} r^{\alpha / p-1}\left|a_{r}(f)\right|\right) .
\end{aligned}
$$

Further, we apply the following Hardy-type inequality (see e.g. [Le 1$]$, [Po]): for $\left\{a_{n} \geq 0\right\}$ and $\left\{\lambda_{n}>0\right\}$,

$$
\sum_{n=1}^{\infty} \lambda_{n}\left(\sum_{\nu=1}^{n} a_{\nu}\right)^{p} \leq p^{p} \sum_{n=1}^{\infty} \lambda_{n}^{1-p} a_{n}^{p}\left(\sum_{\nu=n}^{\infty} \lambda_{\nu}\right)^{p} \quad(p \geq 1) .
$$

We obtain 


$$
\begin{aligned}
& \sum_{n=1}^{\infty} n^{p-2}\left(n^{\frac{\alpha}{p}-1} \sum_{r=0}^{[n / 2]}\left|a_{r}(f)\right|\right)^{p} \\
= & \sum_{n=1}^{\infty} n^{\alpha-2}\left(\sum_{r=0}^{[n / 2]}\left|a_{r}(f)\right|\right)^{p} \leq C(p) \sum_{n=1}^{\infty} n^{(\alpha-2)(1-p)}\left|a_{n}(f)\right|^{p}\left(\sum_{r=n}^{\infty} r^{\alpha-2}\right)^{p} \\
\leq & C(p, \alpha) \sum_{n=1}^{\infty} n^{\alpha+p-2}\left|a_{n}(f)\right|^{p} .
\end{aligned}
$$

Let us now take any $\gamma \in(1-\alpha, 1)$, for instance, $\gamma=(2-\alpha) / 2$. Then, by Hölder's inequality, we get

$$
\sum_{n=1}^{\infty} n^{p-2}\left(\sum_{r=[n / 2]+1}^{n}(n-r+1)^{\alpha / p-1}\left|a_{r}(f)\right|\right)^{p}
$$$$
=\sum_{n=1}^{\infty} n^{p-2}\left(\sum_{r=[n / 2]+1}^{n}(n-r+1)^{(\alpha+\gamma-p) / p}(n-r+1)^{-\gamma / p}\left|a_{r}(f)\right|\right)^{p}
$$$$
\leq \sum_{n=1}^{\infty} n^{p-2} \sum_{r=[n / 2]+1}^{n}(n-r+1)^{-\gamma}\left|a_{r}(f)\right|^{p}\left(\sum_{r=[n / 2]+1}^{n}(n-r+1)^{\frac{\alpha+\gamma-p}{p-1}}\right)^{p-1}
$$$$
\leq C(p, \alpha) \sum_{n=1}^{\infty} n^{p-3+\alpha+\gamma} \sum_{r=[n / 2]+1}^{n}(n-r+1)^{-\gamma}\left|a_{r}(f)\right|^{p}
$$$$
=C(p, \alpha) \sum_{r=1}^{\infty}\left|a_{r}(f)\right|^{p} \sum_{n=r}^{2 r}(n-r+1)^{-\gamma} n^{p-3+\alpha+\gamma}
$$$$
\leq C(p, \alpha) \sum_{r=1}^{\infty}\left|a_{r}(f)\right|^{p} r^{\alpha+p-2} .
$$

Similarly,

$$
\begin{aligned}
\sum_{n=1}^{\infty} n^{p-2} & \left(\sum_{r=n+1}^{2 n}(r-n)^{\alpha / p-1}\left|a_{r}(f)\right|\right)^{p} \\
& \leq \sum_{n=1}^{\infty} n^{p-2} \sum_{r=n+1}^{2 n}(r-n)^{-\gamma}\left|a_{r}(f)\right|^{p}\left(\sum_{r=n+1}^{2 n}(r-n)^{\frac{\alpha+\gamma-p}{p-1}}\right)^{p-1} \\
& \leq C(p, \alpha) \sum_{r=1}^{\infty}\left|a_{r}(f)\right|^{p} r^{\alpha+p-2} .
\end{aligned}
$$

To estimate the last term in (3.3), we apply Hardy's inequality dual to (3.4): for $\left\{a_{n} \geq 0\right\}$ and $\left\{\lambda_{n}>0\right\}$, 


$$
\sum_{n=1}^{\infty} \lambda_{n}\left(\sum_{\nu=n}^{\infty} a_{\nu}\right)^{p} \leq p^{p} \sum_{n=1}^{\infty} \lambda_{n}^{1-p} a_{n}^{p}\left(\sum_{\nu=1}^{n} \lambda_{\nu}\right)^{p} \quad(p \geq 1)
$$

Then

$$
\begin{aligned}
\sum_{n=1}^{\infty} n^{p-2}\left(\sum_{r=2 n+1}^{\infty} r^{\alpha / p-1}\left|a_{r}(f)\right|\right)^{p} & \\
& \leq C(p) \sum_{n=1}^{\infty} n^{(p-2)(1-p)} n^{\alpha-p}\left|a_{n}(f)\right|^{p}\left(\sum_{r=1}^{n} r^{p-2}\right)^{p} \\
& \leq C_{1}(p) \sum_{n=1}^{\infty} n^{\alpha+p-2}\left|a_{n}(f)\right|^{p} .
\end{aligned}
$$

Collecting (3.3), (3.5)-(3.7), and (3.9), we get $\sum_{n=1}^{\infty} n^{p-2}\left|a_{n}(h)\right|^{p}<\infty$, and the statement of Theorem 4.1 follows from Paley's theorem.

Let now $p \in(1,2)$. We are going to show that condition (3.1) implies $\int|h(x)|^{p} x^{p-2} d x<\infty, \quad h(x)=f(x) \varphi(x), \quad \varphi(x)=x^{-\beta / p}, \quad \beta=\alpha+p-2$, where $0<\beta<p-1$.

Note that $\varphi(x) \in L_{p^{\prime}}$ and

$$
\sum_{n}\left|a_{n}(f)\right|^{2} \leq\left(\sum_{n}\left|a_{n}(f)\right|^{p} n^{\alpha+p-2}\right)^{2 / p},
$$

i.e., $f \in L_{2}$ and therefore $f \in L_{p}$. Then Hölder's inequality gives $h \in L_{1}$. Therefore, by classical Paley's theorem, it is enough to show that

$$
\sum_{n}\left|a_{n}(h)\right|^{p}<\infty
$$

The rest of the proof is similar to the case $p>2$. We use relations (3.2)(3.3) with $\beta$ in place of $\alpha$. To estimate the first and the last terms, we apply Hardy's inequalities (3.4) and (3.8). To estimate the middle terms, we make use of Hölder's inequality with parameter $\gamma=(2-\beta) / \beta$ (note that $0<\beta<p-1<1$ ). Finally, we get

$$
\sum_{n}\left|a_{n}(h)\right|^{p} \leq C \sum_{n} n^{\beta}\left|a_{n}(f)\right|^{p}, \quad 0<\beta<p-1,
$$

as desired. The proof is now complete.

REMARK 3.2. For $\alpha<\max (0,2-p)$ the statement of the previous theorem does not hold.

Indeed, for $p \geq 2$, defining the series

$$
f(x)=\sum_{n=1}^{\infty}(-1)^{n} n^{1 / p-1} \cos n x,
$$


we note that $\alpha<0$ and

$$
\sum_{n=1}^{\infty} n^{\alpha+p-2}\left|a_{n}\right|^{p}=\sum_{n=1}^{\infty} n^{-|\alpha|-1}<\infty .
$$

On the other hand, since $f(x) \sim(\pi-x)^{-1 / p}$, we get $|f(x)|^{p} x^{-\alpha} \notin L(0, \pi)$.

Let now $1<p<2$ and put $\beta:=\alpha+p-2<0$. In this case we consider

$$
\sum_{k=2}^{\infty} \frac{1}{\ln ^{2} k} e^{i k x}
$$

It is known that this series converges in $L(0, \pi)$ and diverges in $L_{p}(0,1)$ ([Zy, Ch. V; Ch. XII]). Thus for any integer $l$ one can find integers $N_{l}<M_{l}$ such that

$$
S_{l}(x)=\sum_{k=N_{l}}^{M_{l}} \frac{1}{\ln ^{2} k} e^{i k x}
$$

and $\left\|S_{l}\right\|_{1}<1 / l^{2}$ and $\left\|S_{l}\right\|_{p}>l^{2}$. Let us define

$$
c_{l}:=\sum_{k=N_{l}}^{M_{l}} \frac{1}{\ln ^{2 p} k} .
$$

Now we choose integers $R_{l}$ such that

$$
R_{l}+N_{l}>R_{l-1}+M_{l-1} \quad \text { and } \quad R_{l}^{\beta} c_{l}<\frac{1}{l^{2}} \quad \text { for } l=2,3, \ldots
$$

Then the series

$$
\sum_{l=1}^{\infty} e^{i R_{l} x} S_{l}(\pi-x) \equiv \sum_{l=1}^{\infty} e^{i R_{l} x} \sum_{k=N_{l}}^{M_{l}} \frac{e^{i k(\pi-x)}}{\ln ^{2} k}
$$

converges to a function $f(x)$ in $L(0, \pi)$ and

$$
\sum_{n=1}^{\infty} n^{\beta}\left|a_{n}(f)\right|^{p} \leq C \sum_{l=1}^{\infty} R_{l}^{\beta} c_{l}<\infty .
$$

On the other hand, $f(x) \notin L_{p}(\pi-1, \pi)$ (since the sequence of partial sums of $(3.11)$ diverges in $\left.L_{p}(\pi-1, \pi)\right)$, and thus $|f(x)|^{p} x^{-\alpha} \notin L(0, \pi)$.

4. Weighted $L_{p}$-integrability $(1 \leq p<\infty)$ for the series with monotone type coefficients. In this section we are going to study the $L_{p^{-}}$-integrability problems with power weights $x^{-\alpha}$ for trigonometric series (1.1) and (1.2) with regularity conditions on the coefficients. Theorem 2.4 and Remark 3.2 show that in order to obtain any positive result of the type

$$
\sum_{n=1}^{\infty} n^{\alpha+p-2}\left|a_{n}(f)\right|^{p}<\infty \Rightarrow|f(x)|^{p} x^{-\alpha} \in L(0, \pi)
$$


for $p=1$ or to extend the range $\max (0,2-p) \leq \alpha<1$ for $1<p<\infty$, one has to impose some additional conditions on $\left\{a_{n}\right\}$. Note here that since we want to consider a constant function as an example of $f(x)$, we have to assume $\alpha<1$. For odd functions, generally, it is possible to consider $\alpha<\max (0,2-p)$ as well as $\alpha \geq 1$.

Typically many authors considered series with monotone (or quasi-monotone) decreasing coefficients. We would like to extend the following result.

TheOREM 4.1. Let $\left\{a_{n}, b_{n} \geq 0\right\} \in \mathfrak{M}, 1<p<\infty$ and $1-p<\alpha<1$. Then

$$
\frac{|\psi(x)|^{p}}{x^{\alpha}} \in L(0, \pi) \Leftrightarrow \sum_{n=1}^{\infty} n^{\alpha+p-2} \lambda_{n}^{p}<\infty,
$$

where $\psi(x)$ is either $f(x)$ or $g(x)$ and $\lambda_{n}$ is either $a_{n}$ or $b_{n}$, respectively.

In the case when $\mathfrak{M}$ denotes the class $M$ of all decreasing sequences, this theorem was proved in $[\mathrm{Bo}],\left[\mathrm{He}_{1}\right]$; for $\mathfrak{M}=Q M$, the class of quasi-monotone sequences, in $[\mathrm{AW}]$; for $\mathfrak{M}=\operatorname{GM}(\bar{\beta})$ in $\left[\mathrm{Ti}_{1}\right]$; and for $\mathfrak{M}=\operatorname{GM}\left(\beta^{*}\right)$ in $[\mathrm{YZZ}]$, where

$$
\operatorname{GM}(\beta):=\left\{\left\{a_{k}\right\}: \sum_{k=n}^{2 n}\left|\Delta a_{k}\right| \leq C \beta_{n}\right\}
$$

and

$$
\bar{\beta}_{n}=\left|a_{n}\right|, \quad \beta_{n}^{*}=\sum_{k=[n / c]}^{[n c]} \frac{\left|a_{k}\right|}{k} \quad \text { for some } c>1 .
$$

Note that $\left(\left[\mathrm{Ti}_{1}\right],\left[\mathrm{Ti}_{3}\right]\right)$

$$
M \subsetneq Q M \subsetneq \operatorname{GM}(\bar{\beta}) \subsetneq \operatorname{GM}\left(\beta^{*}\right) .
$$

We would like to consider $\beta$-generally monotone coefficients. Let $\theta \in$ $(0,1]$. By definition, $\overline{\mathrm{GM}}_{\theta}$ is the class $\overline{\mathrm{GM}}(\beta)$ with

$$
\beta_{n}=n^{\theta-1} \sum_{k=[n / c]}^{\infty} \frac{\left|a_{k}\right|}{k^{\theta}} \quad \text { for some } c>1 .
$$

In other words, $\overline{\mathrm{GM}}_{\theta}$ is the collection of all sequences such that

$$
\sum_{k=n}^{\infty}\left|\Delta a_{k}\right|<C n^{\theta-1} \sum_{k=[n / c]}^{\infty} \frac{\left|a_{k}\right|}{k^{\theta}}<\infty \quad \text { for some } c>1 .
$$

For $\theta=1$ we define $\overline{\mathrm{GM}} \equiv \overline{\mathrm{GM}}_{1}$.

We have (cf. (4.1))

$$
\mathrm{GM}\left(\beta^{*}\right) \subsetneq \overline{\mathrm{GM}} \equiv \overline{\mathrm{GM}}_{1} \subseteq \overline{\mathrm{GM}}_{\theta_{2}} \subseteq \overline{\mathrm{GM}}_{\theta_{1}}, \quad 0<\theta_{1} \leq \theta_{2} \leq 1 .
$$

The fact that $\operatorname{GM}\left(\beta^{*}\right) \subseteq \overline{\mathrm{GM}}$ is clear and an example of a sequence such that $\overline{\mathrm{GM}} \backslash \operatorname{GM}\left(\beta^{*}\right) \neq \emptyset$ will be constructed in the last section. 
Our main results in this section are the following:

Theorem 4.2. (Sine) Let $\left\{b_{n} \geq 0\right\} \in \overline{\mathrm{GM}}_{\theta}, \theta \in(0,1], 1 \leq p<\infty$. If

$$
1-\theta p<\alpha<1+p
$$

then

$$
\frac{|g(x)|^{p}}{x^{\alpha}} \in L(0, \pi) \Leftrightarrow \sum_{n=1}^{\infty} n^{\alpha+p-2} b_{n}^{p}<\infty .
$$

Theorem 4.3. (Cosine) Let $\left\{a_{n} \geq 0\right\} \in \overline{\mathrm{GM}}_{\theta}, \theta \in(0,1], 1 \leq p<\infty$. If

$$
1-\theta p<\alpha<1
$$

then

$$
\frac{|f(x)|^{p}}{x^{\alpha}} \in L(0, \pi) \Leftrightarrow \sum_{n=1}^{\infty} n^{\alpha+p-2} a_{n}^{p}<\infty .
$$

REMARK 4.4. We note that the condition

$$
\sum_{n=1}^{\infty} n^{\alpha+p-2} \lambda_{n}^{p}<\infty, \quad 1 \leq p<\infty, \quad \lambda_{k} \geq 0,
$$

always implies (by Hölder's inequality) the condition

$$
\sum_{k=1}^{\infty} \frac{\lambda_{k}}{k^{\theta}}<\infty \quad \text { for } \theta \in(0,1], \alpha>1-\theta p .
$$

Proof of Theorem 4.2. Let $x \in(\pi /(n+1), \pi / n]$. Then, by Abel's transform,

$$
\begin{aligned}
|g(x)| & \leq x \sum_{k=1}^{n} k b_{k}+\left|\sum_{k=n+1}^{\infty} b_{k} \sin k x\right| \\
& \leq x \sum_{k=1}^{n} k b_{k}+\sum_{k=n}^{\infty}\left|\left(b_{k}-b_{k+1}\right)\left(\widetilde{D}_{k}(x)-\widetilde{D}_{n}(x)\right)\right|,
\end{aligned}
$$

where $\widetilde{D}_{k}(x):=\sum_{n=1}^{k} \sin n x$ for $k \in \mathbb{N}$. Since $\left|\widetilde{D}_{k}(x)\right|=O(1 / x)$ and $\left\{a_{n}\right\} \in$ $\overline{\mathrm{GM}}_{\theta}$, we get

$$
|g(x)| \leq C\left(x \sum_{k=1}^{n} k b_{k}+n \sum_{k=n}^{\infty}\left|b_{k}-b_{k+1}\right|\right) \leq C\left(x \sum_{k=1}^{n} k b_{k}+n^{\theta} \sum_{k=[n / c]}^{\infty} \frac{\left|b_{k}\right|}{k^{\theta}}\right) .
$$

Hence,

$$
|g(x)| \leq C\left(x \sum_{k=1}^{n} k\left|b_{k}\right|+n^{\theta} \sum_{k=n}^{\infty} \frac{\left|b_{k}\right|}{k^{\theta}}\right) .
$$


Further (define $\gamma(x)=x^{-\alpha}$ and $\gamma_{n}=n^{\alpha}$ ), we have

$$
\begin{aligned}
& \int_{0}^{\pi} \gamma(x)|g(x)|^{p} d x=\sum_{n=1}^{\infty} \int_{\pi /(n+1)}^{\pi / n} \gamma(x)|g(x)|^{p} d x \\
& \leq C(p, \alpha) \sum_{n=1}^{\infty} \frac{\gamma_{n}}{n^{2+p}}\left(\sum_{k=1}^{n} k\left|b_{k}\right|\right)^{p}+C(p, \alpha) \sum_{n=1}^{\infty} \frac{n^{\theta p} \gamma_{n}}{n^{2}}\left(\sum_{k=n}^{\infty} \frac{\left|b_{k}\right|}{k^{\theta}}\right)^{p} .
\end{aligned}
$$

By Hardy's inequalities (3.4) and (3.8), we obtain

$$
\int_{0}^{\pi} \gamma(x)|g(x)|^{p} d x \leq C(p, \alpha) \sum_{n=1}^{\infty} \gamma_{n} n^{p-2}\left|b_{n}\right|^{p}
$$

for $1-\theta p<\alpha<1+p$.

Let us now prove the " $\Rightarrow$ " part. Note that if $1-p<\alpha$, then $g(x) \in$ $L(0, \pi)$. Integrating $g(x)$, we have

$$
F(x):=\int_{0}^{x} g(t) d t=\sum_{n=1}^{\infty} \frac{b_{n}}{n}(1-\cos n x)=2 \sum_{n=1}^{\infty} \frac{b_{n}}{n} \sin ^{2} \frac{n x}{2} .
$$

Since $\left\{b_{n} \geq 0\right\}$, this gives

$$
F\left(\frac{\pi}{k}\right) \geq C \sum_{n=[k / 2]}^{k} \frac{b_{n}}{n} .
$$

As the sequence $\left\{b_{s}\right\}$ is generally monotone, we estimate

$$
\begin{aligned}
b_{s} & \leq \sum_{l=s}^{\infty}\left|\Delta b_{l}\right| \leq C s^{\theta-1} \sum_{l=[s / c]}^{\infty} \frac{b_{l}}{l^{\theta}} \leq C s^{\theta-1} \sum_{l=[s / c]}^{\infty} \frac{1}{l^{\theta}} \sum_{n=[l / 2]}^{l} \frac{b_{n}}{n} \\
& \leq C s^{\theta-1} \sum_{l=[s / c]}^{\infty} \frac{1}{l^{\theta}} F\left(\frac{\pi}{l}\right) .
\end{aligned}
$$

Applying this, we get

$$
J:=\sum_{k=1}^{\infty} \gamma_{k} k^{p-2} b_{k}^{p} \leq C(p) \sum_{k=1}^{\infty} \gamma_{k} k^{p-2} k^{(\theta-1) p}\left(\sum_{\nu=[k / c]}^{\infty} \frac{1}{\nu^{\theta}} F\left(\frac{\pi}{\nu}\right)\right)^{p} .
$$

Further, because $\gamma_{[c n]} \asymp \gamma_{n}$ for $c>0$, we use inequality (3.8):

$$
\begin{aligned}
J & \leq C(p) \sum_{k=1}^{\infty} \gamma_{k} k^{p-2} k^{(\theta-1) p}\left(\sum_{\nu=k}^{\infty} \frac{1}{\nu^{\theta}} F\left(\frac{\pi}{\nu}\right)\right)^{p} \\
& \leq C(p) \sum_{\nu=1}^{\infty} \frac{F^{p}(\pi / \nu)}{\nu^{\theta p}}\left(\gamma_{\nu} \nu^{\theta p-2}\right)^{1-p}\left\{\sum_{k=1}^{\nu} \gamma_{k} k^{\theta p-2}\right\}^{p} .
\end{aligned}
$$


Since $\alpha>1-\theta p$, we obtain

$$
\begin{aligned}
J & \leq C(p) \sum_{\nu=1}^{\infty} \frac{F^{p}(\pi / \nu)}{\nu^{\theta p}}\left(\gamma_{\nu} \nu^{\theta p-2}\right)^{1-p}\left\{\sum_{k=1}^{\nu} \gamma_{k} k^{\theta p-2}\right\}^{p} \\
& \leq C(p) \sum_{\nu=1}^{\infty} F^{p}\left(\frac{\pi}{\nu}\right)\left(\gamma_{\nu} \nu^{p-2}\right) .
\end{aligned}
$$

Defining $d_{\nu}:=\int_{\pi /(\nu+1)}^{\pi / \nu}|g(x)| d x(\nu \in \mathbb{N})$, we immediately get

$$
F\left(\frac{\pi}{k}\right) \leq \sum_{\nu=k}^{\infty} d_{\nu}
$$

Hence

$$
J \leq C(p) \sum_{\nu=1}^{\infty} F^{p}\left(\frac{\pi}{\nu}\right)\left(\gamma_{\nu} \nu^{p-2}\right) \leq C(p) \sum_{\nu=1}^{\infty}\left(\gamma_{\nu} \nu^{p-2}\right)\left(\sum_{s=\nu}^{\infty} d_{s}\right)^{p} .
$$

Using (3.8) for $\alpha>1-\theta p \geq 1-p$, we obtain

$$
J \leq C(p) \sum_{\nu=1}^{\infty} \gamma_{\nu} \nu^{2 p-2} d_{\nu}^{p}
$$

By Hölder's inequality $\left(p \in(1, \infty), p^{\prime}=p /(p-1)\right)$,

$$
\begin{aligned}
d_{\nu}^{p} & =\left(\int_{\pi /(\nu+1)}^{\pi / \nu}|g(x)| d x\right)^{p} \leq C(p) \int_{\pi /(\nu+1)}^{\pi / \nu}|g(x)|^{p} d x\left(\frac{1}{\nu^{2}}\right)^{p / p^{\prime}} \\
& =C(p) \nu^{2(1-p)} \int_{\pi /(\nu+1)}^{\pi / \nu}|g(x)|^{p} d x .
\end{aligned}
$$

Finally,

$$
\sum_{k=1}^{\infty} \gamma_{k} k^{p-2} b_{k}^{p} \leq C(p, \alpha) \int_{0}^{\pi}|g(x)|^{p} \gamma(x) d x
$$

which completes the proof.

Proof of Theorem 4.3. First we remark

$$
\frac{|g(x)|^{p}}{x^{\alpha}} \in L(0, \pi) \Leftrightarrow \int_{[-\pi, \pi]}|g(x)|^{p}|x|^{-\alpha} d x<\infty .
$$

It was shown in $[\mathrm{Bab}]$ that

$$
\int_{[-\pi, \pi]}|\widetilde{g(x)}|^{p}|x|^{-\alpha} d x \leq C(p, \alpha) \int_{[-\pi, \pi]}|g(x)|^{p}|x|^{-\alpha} d x
$$

for $-1<-\alpha<p-1$. A more general result was obtained in [HMW] for the $A_{p}$-Muckenhoupt weights (note that $|x|^{-\alpha}$ is an $A_{p}$-weight if and only if 
$-1<-\alpha<p-1)$ : for $\omega \in A_{p}$ we have

$$
\int_{[-\pi, \pi]}|\widetilde{g(x)}|^{p} \omega(x) d x \leq C(p, \omega) \int_{[-\pi, \pi]}|g(x)|^{p} \omega(x) d x .
$$

Using these inequalities twice with $-1<-\alpha<\theta p-1$ (note that $\theta p-1<$ $p-1$ ), we get

$$
\begin{aligned}
\int_{[-\pi, \pi]}|g(x)|^{p}|x|^{-\alpha} d x & \leq C(p, \alpha) \int_{[-\pi, \pi]}|f(x)|^{p}|x|^{-\alpha} d x \\
& \leq C(p, \alpha) \int_{[-\pi, \pi]}|g(x)|^{p}|x|^{-\alpha} d x
\end{aligned}
$$

Since

$$
-1<-\alpha<\theta p-1 \Leftrightarrow 1-\theta p<\alpha<1,
$$

the proof of the theorem is finished.

5. Uniform convergence. First, let us consider the case of the cosine series. One has the following results. If either

(1) $a_{n} \geq 0$, or

(2) $\left\{a_{n}\right\} \in \operatorname{GM}(\beta)$ and $n \beta_{n}=o(1)$ as $n \rightarrow \infty$, then

series (1.1) converges uniformly on $[0,2 \pi] \Leftrightarrow \sum_{n} a_{n}$ converges.

In the case when $a_{n} \geq 0$ this criterion is clear; for the series with $\beta$-generally monotone coefficients it was proved in $\left[\mathrm{DT}_{1}\right]$.

For the sine series, Chaundy and Joliffe ([CJ], [Zy, Vol. 1, p. 183]) proved the following: a necessary and sufficient condition for series (1.2), where $b_{n} \geq b_{n+1} \geq \cdots$, to be uniformly convergent on $[0,2 \pi]$ is $n b_{n} \rightarrow 0$ as $n \rightarrow \infty$.

We remark that this problem has been extensively studied recently. For instance, series with $\beta$-generally monotone coefficients were considered for $\bar{\beta}_{n}=\left|a_{n}\right|, \widetilde{\beta}_{n}=\left|a_{n}\right|+\cdots+\left|a_{n+N}\right|, \beta_{n}^{*}=\sum_{k=[n / c]}^{[c n]}\left|a_{k}\right| / k$ (see the history of the question in $\left.\left[\mathrm{Ti}_{3}\right]\right)$. We note that $\operatorname{GM}\left(\beta^{*}\right)$ is the largest known class for which Chaundy-Joliffe's criterion holds true.

Theorem 5.1. Let $b \in \operatorname{GM}(\beta)$.
(A) If

$$
\sum_{k=n}^{\infty}\left|\Delta b_{k}\right|=o(1 / n)
$$


as $n \rightarrow \infty$, then series (1.2) converges uniformly on $[0,2 \pi]$ and

$$
\left\|g(x)-S_{n}(g, x)\right\|_{\infty} \leq C \max _{\nu \geq n} \nu \sum_{k=\nu}^{\infty}\left|\Delta b_{k}\right| .
$$

(B) Let a non-negative sequence $b=\left\{b_{n}\right\}$ satisfy

$$
b_{n} \leq \frac{C}{n} \max _{k \geq[n / c]} \sum_{s=k}^{2 k} b_{s} \quad \text { for some } c>1 .
$$

Then the uniform convergence of series (1.2) implies

$$
n b_{n}=o(1) \quad \text { as } n \rightarrow \infty .
$$

Even though the proof of this theorem is relatively straightforward, both statements are sharp.

REMARK 5.2. First, in (5.1) we cannot substitute $O(1 / n)$ for $o(1 / n)$. According to (B), we have the following result. For any non-decreasing positive sequence $\{\varphi(n)\}_{n \in \mathbb{N}}$ satisfying $\varphi(n) \rightarrow \infty$ as $n \rightarrow \infty$, there exists an odd function $g(x) \in C([0, \pi])$ with uniformly convergent Fourier series such that its sine coefficients are non-negative and satisfy

$$
b_{n} \leq C \frac{\varphi(n)}{n} \max _{k \geq[n / c]} \sum_{s=k}^{2 k} b_{s} \quad \text { for some } c>1
$$

for all $n$, but $n b_{n} \nrightarrow 0$ as $n \rightarrow \infty$.

Let us now present a generalization of Chaundy-Joliffe's criterion as well as its extensions.

Corollary 5.3. Let $\left\{b_{n} \geq 0\right\} \in \operatorname{GM}(\bar{\beta})$, where

$$
\bar{\beta}_{n}=\frac{1}{n} \max _{k \geq[n / c]} \sum_{s=k}^{2 k} b_{k} \quad \text { for some } c>1 .
$$

Then series (1.2) converges uniformly on $[0,2 \pi]$ if and only if condition (5.3) holds.

REMARK 5.4. We have $\operatorname{GM}\left(\beta^{*}\right) \subsetneq \operatorname{GM}(\bar{\beta})$, where

$$
\beta_{n}^{*}=\sum_{k=[n / c]}^{[c n]} \frac{\left|b_{k}\right|}{k} \quad \text { and } \quad \bar{\beta}_{n}=\frac{1}{n} \max _{k \geq[n / c]} \sum_{s=k}^{2 k} b_{k} \quad \text { for some } c>1 .
$$

This remark will be proved in the last section.

Proof of Theorem 5.1. Item (A) was actually proved in [DT 1 , Th. 2.1]. To prove the second part, if series (1.2) with non-negative coefficients converges 
uniformly, then

$$
\frac{1}{n} \sum_{k=1}^{n} k b_{k}=o(1)
$$

This gives

$$
\sum_{k=n}^{2 n} b_{k}=o(1)
$$

Further, by (5.2), we get

$$
n b_{n} \leq C \max _{k \geq[n / c]} \sum_{s=k}^{2 k} b_{k}=o(1) \quad \text { as } n \rightarrow \infty,
$$

that is, condition (5.3) is satisfied.

Proof of Remark 5.2. First, we consider the sine series

$$
\sum_{n} \frac{\sin n x}{n}
$$

Here the condition $\sum_{k=n}^{\infty}\left|\Delta b_{k}\right|=O(1 / n)$ holds but the series does not converge uniformly on $[0,2 \pi]$.

Let us now prove the second part. Without loss of generality, we assume that $\varphi(n) \leq \ln (n+1), \varphi(2 n) \leq 2 \varphi(n)$ as $n \geq 1$ and $\varphi(1) \geq 1$. Define $\psi(n):=\sqrt{\varphi([n / 2])}$ for $n \geq 2$ and $\psi(1):=\frac{1}{2} \sqrt{\varphi(1)}$.

Let $b_{n, 1}:=1 / n \psi(n)$ for $n \geq 1$. Then for any $n$ we have

$$
\frac{4}{n} \sum_{k=n+1}^{2 n} b_{k, 1} \geq \frac{2}{n \psi(2 n)} \geq b_{n, 1} .
$$

We next define the sequence

$$
b_{n, 2}:= \begin{cases}2^{-k} \psi\left(2^{k}\right) & \text { for } n=2^{k}, k=1,2, \ldots, \\ 0 & \text { otherwise. }\end{cases}
$$

For any $k$, according to (5.4) we have

$$
\frac{4 \varphi\left(2^{k}\right)}{2^{k}} \sum_{k=2^{k}+1}^{2^{k+1}} b_{k, 1} \geq \frac{\varphi\left(2^{k}\right)}{2^{k} \psi\left(2^{k}\right)} \geq b_{2^{k}, 2} .
$$

Inequalities (5.4) and (5.5) imply that the sequence $b_{n}=b_{n, 1}+b_{n, 2}$ satisfies

$$
b_{n} \leq 8 \frac{\varphi(n)}{n} \sum_{k=n+1}^{2 n} b_{k} \leq 8 \frac{\varphi(n)}{n} \max _{k \geq[n / c]} \sum_{s=k}^{2 k} b_{k} .
$$

This is the sequence of Fourier coefficients of the function $g(x)=g_{1}(x)+$ 
$g_{2}(x)$, where

$$
g_{i}(x)=\sum_{n=1}^{\infty} b_{n, i} \sin n x \quad \text { for } i=1,2 .
$$

The function $g_{1}(x)$ is the sum of a sine series with monotone coefficients $\left\{b_{n, 1}\right\}$ such that $n b_{n, 1} \rightarrow 0$ as $n \rightarrow \infty$. By the Chaundy-Joliffe theorem, $g_{1}(x) \in C([0, \pi])$ and series (5.6) converges uniformly. As regards $g_{2}(x)$, it is the sum of an absolutely convergent series and therefore it is continuous. Then the series $\sum_{n=1}^{\infty} b_{n} \sin n x$ converges uniformly. Finally, we remark that $n b_{n, 2} \nrightarrow 0$ as $n \rightarrow \infty$.

Proof of Corollary 5.3. First, condition (5.3) implies $\sum_{k=n}^{2 n} b_{k}=o(1)$. Since $\left\{b_{n}\right\} \in \operatorname{GM}(\bar{\beta})$, we have

$$
\begin{aligned}
\sum_{k=n}^{\infty}\left|\Delta b_{k}\right| & \leq 2 \sum_{k=[n / 2]}^{\infty} \frac{1}{k} \sum_{s=k}^{2 k-1}\left|\Delta b_{s}\right| \\
& \leq C\left(\max _{k \geq[n / c]} \sum_{s=k}^{2 k} b_{k}\right) \sum_{k=[n / 2]}^{\infty} \frac{1}{k^{2}} \leq \frac{C}{n} \max _{k \geq[n / c]} \sum_{s=k}^{2 k} b_{k} .
\end{aligned}
$$

Combining this with $\sum_{k=n}^{2 n} b_{k}=o(1)$, we arrive at (5.2). Then from Theorem 5.1 (A) series (1.2) converges uniformly on $[0,2 \pi]$.

Conversely, using

$$
b_{n} \leq \sum_{k=n}^{\infty}\left|\Delta b_{k}\right| \leq \frac{C}{n} \max _{k \geq[n / c]} \sum_{s=k}^{2 k} b_{k},
$$

we apply Theorem 5.1(B). Thus (5.3) follows.

6. Applications to approximation theory. Let $f \in C([0,2 \pi])$ and let $E_{n}(f)$ be the best approximation of $f$ by trigonometric polynomials of order $n$. Let also $\omega_{k}(f, t)$ be the modulus of smoothness of $f$ of order $k>0$, i.e.,

$$
\omega_{k}(f, t)=\sup _{|h| \leq t}\left\|\sum_{\nu=0}^{\infty}(-1)^{\nu}\left(\begin{array}{l}
k \\
\nu
\end{array}\right) f(x+(k-\nu) h)\right\|,
$$

where $\left(\begin{array}{l}k \\ \nu\end{array}\right)=k(k-1) \cdots(k-\nu+1) / \nu$ ! for $\nu \geq 1,\left(\begin{array}{l}k \\ \nu\end{array}\right)=1$ for $\nu=0$, and $\|f(\cdot)\|=\max _{x \in[0,2 \pi]}|f(x)|$.

It is well known (see, e.g., [DL, pp. 205, 208]) that the best approximation and the modulus of smoothness are related as follows:

$$
C(k) E_{n}(f) \leq \omega_{k}\left(f, \frac{1}{n}\right) \leq C(k) \frac{1}{n^{k}} \sum_{\nu=0}^{n-1}(\nu+1)^{k-1} E_{\nu}(f) .
$$


Our main goal in this section is to study classes of trigonometric series for which one can check the sharpness of the left-hand side inequality (Jacksontype estimate) and the right-hand side inequality (Bernstein-Stechkin type estimate). For the history of this question we recommend $\left[\mathrm{Ti}_{3}\right]$.

As in Section 5, we will deal with a sequence $\left\{d_{n}\right\} \in \operatorname{GM}(\bar{\beta})$, i.e.,

$$
\sum_{k=n}^{2 n-1}\left|\Delta d_{k}\right| \leq \frac{C}{n} \max _{k \geq[n / c]} \sum_{s=k}^{2 k}\left|d_{s}\right| \quad \text { for some } c>1 .
$$

Theorem 6.1. (Cosine, sine) Let $a=\left\{a_{n}\right\}_{n=1}^{\infty}, b=\left\{b_{n}\right\}_{n=1}^{\infty} \in \operatorname{GM}(\bar{\beta})$ be non-negative sequences. Then

$$
\begin{array}{ll}
\omega_{k}\left(f, \frac{1}{n}\right) \asymp n^{-k} \sum_{\nu=0}^{n-1}(\nu+1)^{k-1} E_{\nu}(f) & \text { for } k \neq 2 l-1(l \in \mathbb{N}), \\
\omega_{k}\left(f, \frac{1}{n}\right) \asymp n^{-k} \max _{\nu \in[0, n]}(\nu+1)^{k} E_{\nu}(f) & \text { for } k=2 l-1(l \in \mathbb{N}), \\
\omega_{k}\left(g, \frac{1}{n}\right) \asymp n^{-k} \sum_{\nu=0}^{n-1}(\nu+1)^{k-1} E_{\nu}(g) & \text { for } k \neq 2 l(l \in \mathbb{N}), \\
\omega_{k}\left(g, \frac{1}{n}\right) \asymp n^{-k} \max _{\nu \in[0, n]}(\nu+1)^{k} E_{\nu}(g) & \text { for } k=2 l(l \in \mathbb{N}) .
\end{array}
$$

Theorem 6.2. (Cosine, sine) Let $a=\left\{a_{n}\right\}_{n=1}^{\infty}, b=\left\{b_{n}\right\}_{n=1}^{\infty} \in \operatorname{GM}(\bar{\beta})$ be non-negative sequences. Then for any $\varepsilon>0$ we have

$$
\begin{array}{ll}
\omega_{k}\left(f, \frac{1}{n}\right) \asymp n^{-k} \sum_{\nu=1}^{n} \nu^{k-1} \omega_{k+\varepsilon}\left(f, \frac{1}{\nu}\right) & \text { for } k \neq 2 l-1(l \in \mathbb{N}), \\
\omega_{k}\left(f, \frac{1}{n}\right) \asymp n^{-k} \max _{\nu \in[1, n]} \nu^{k} \omega_{k+\varepsilon}\left(f, \frac{1}{\nu}\right) \quad \text { for } k=2 l-1(l \in \mathbb{N}), \\
\omega_{k}\left(g, \frac{1}{n}\right) \asymp n^{-k} \sum_{\nu=1}^{n} \nu^{k-1} \omega_{k+\varepsilon}\left(g, \frac{1}{\nu}\right) \quad \text { for } k \neq 2 l(l \in \mathbb{N}), \\
\omega_{k}\left(g, \frac{1}{n}\right) \asymp n^{-k} \max _{\nu \in[1, n]} \nu^{k} \omega_{k+\varepsilon}\left(g, \frac{1}{\nu}\right) \quad \text { for } k=2 l(l \in \mathbb{N}) .
\end{array}
$$

These results were first presented in [Be] for series with quasi-monotone coefficients (without proof). A generalization was given in $\left[\mathrm{Ti}_{3}\right]$. The proofs of Theorems 6.1 and 6.2 can be obtained as in $\left[\mathrm{Ti}_{3}\right]$ and are based on the following theorem. 
Theorem 6.3. (Cosine, sine) Let $a=\left\{a_{n}\right\}_{n=1}^{\infty}, b=\left\{b_{n}\right\}_{n=1}^{\infty} \in \operatorname{GM}(\bar{\beta})$ be non-negative sequences with $\sum_{n} a_{n}<\infty$ and $n b_{n}=o(1)$. Then

$$
\omega_{k}\left(f, \frac{1}{n}\right) \asymp n^{-k} \sum_{m=1}^{n} m^{k} a_{m}+\sum_{m=n}^{\infty} a_{m} \quad \text { for } k \neq 2 l-1(l \in \mathbb{N}),
$$

$$
\omega_{k}\left(f, \frac{1}{n}\right) \asymp n^{-k} \max _{m \in[1, n]} m^{k+1} a_{m}+\sum_{m=n}^{\infty} a_{m} \quad \text { for } k=2 l-1(l \in \mathbb{N}) \text {, }
$$

$$
\begin{array}{ll}
\omega_{k}\left(g, \frac{1}{n}\right) \asymp n^{-k} \sum_{m=1}^{n} m^{k} b_{m}+\max _{m \geq n} m b_{m} & \text { for } k \neq 2 l(l \in \mathbb{N}), \\
\omega_{k}\left(g, \frac{1}{n}\right) \asymp n^{-k} \max _{m \in[1, n]} m^{k+1} b_{m}+\max _{m \geq n} m b_{m} & \text { for } k=2 l(l \in \mathbb{N}) .
\end{array}
$$

Since this theorem actually follows from the results of $\left[\mathrm{Ti}_{3}\right]$ and $\left[\mathrm{Ti}_{2}\right]$, we will only give the proof of (6.8), say.

" $\leq "$. This follows from

$$
\omega_{k}\left(g, \frac{1}{n}\right) \leq C\left(n^{-k} \sum_{m=1}^{n} m^{k}\left|b_{m}\right|+\max _{m \geq n} m\left(\left|b_{m}\right|+\bar{\beta}_{m}\right)\right)
$$

(see $\left[\mathrm{Ti}_{3}\right.$, Th. $\left.4.2(\mathrm{~A})\right]$ ) and the definition of $\bar{\beta}_{m}$.

" $\geq$ ". In $\left[\mathrm{Ti}_{3}\right.$, Th. 4.4(A)] we proved

$$
C \omega_{k}\left(g, \frac{1}{n}\right) \geq n^{-k} \sum_{m=1}^{n} m^{k} b_{m}+\max _{m \geq n} \sum_{\nu=[m / c]}^{[c m]} b_{\nu}, \quad c>1 .
$$

Therefore, since

$$
n\left|b_{n}\right| \leq C \max _{k \geq[n / c]} \sum_{s=k}^{2 k}\left|b_{k}\right|
$$

(see (5.7)), we get

$$
\begin{aligned}
\omega_{k}\left(g, \frac{1}{n}\right) & \geq C\left(n^{-k} \sum_{m=1}^{n} m^{k} b_{m}+\max _{m \geq n} \max _{s \geq[m / c]} \sum_{\nu=s}^{2 s-1} b_{\nu}\right) \\
& \geq C\left(n^{-k} \sum_{m=1}^{n} m^{k} b_{m}+\max _{m \geq n} m b_{m}\right), \quad c>1,
\end{aligned}
$$

and (6.8) follows.

\section{Concluding remarks}

1. The condition $\overline{\mathrm{GM}}_{\theta}$ for $0<\theta<1$ is equivalent to the condition $\operatorname{GM}(\beta)$, where 


$$
\beta_{n} \equiv n^{\theta-1} \sum_{k=[n / c]}^{\infty} \frac{\left|a_{k}\right|}{k^{\theta}} \quad \text { for some } c>1
$$

i.e.,

$$
\sum_{k=n}^{2 n-1}\left|\Delta a_{k}\right| \leq C n^{\theta-1} \sum_{k=[n / c]}^{\infty} \frac{\left|a_{k}\right|}{k^{\theta}} \quad \text { for some } c>1 .
$$

2. Let us construct $\left\{a_{n}\right\} \in \overline{\mathrm{GM}} \equiv \overline{\mathrm{GM}}_{1}$ such that $\left\{a_{n}\right\} \notin \mathrm{GM}\left(\beta^{*}\right)$. Since one clearly has $\overline{\mathrm{GM}} \subseteq \operatorname{GM}(\bar{\beta})$, we immediately prove Remark 5.4 as well.

Set

$$
N_{1}=1, \quad N_{\xi+1}=N_{\xi}+2\left[N_{\xi} \exp \left(N_{\xi}\right)\right] .
$$

Then we define $a=\left\{a_{k}\right\}$ as follows:

$$
a_{k}= \begin{cases}10^{-4}, & 1 \leq k<N_{4}, \\ (-1)^{k} 10^{-\xi}, & N_{\xi} \leq k<2 N_{\xi}, \xi \geq 4, \\ 10^{-\xi}, & 2 N_{\xi} \leq k<2 N_{\xi}+\left[N_{\xi} \exp \left(N_{\xi}\right)\right], \xi \geq 4, \\ 0, & N_{\xi}+\left[N_{\xi} \exp \left(N_{\xi}\right)\right] \leq k<N_{\xi+1}, \xi \geq 4 .\end{cases}
$$

Let us verify that $\left\{a_{n}\right\} \notin \operatorname{GM}\left(\beta^{*}\right)$. For any $k$ we take $s \in \mathbb{N}$ such that $N_{s} \leq k<N_{s+1}$. Then for $k=N_{s}$ we have

$$
\sum_{l=k}^{2 k-1}\left|\Delta a_{l}\right| \geq C \frac{k}{10^{s}} \asymp \frac{N_{s}}{10^{s}} .
$$

But

$$
\sum_{l=[k / c]}^{[c k]} \frac{\left|a_{l}\right|}{l} \leq \frac{C}{10^{s}} \sum_{l=[k / c]}^{[c k]} \frac{1}{l} \asymp \frac{1}{10^{s}}
$$

which contradicts

$$
\sum_{l=k}^{2 k-1}\left|\Delta a_{l}\right| \leq C \sum_{l=[k / c]}^{[c k]} \frac{\left|a_{l}\right|}{l} .
$$

Now we show that $\left\{a_{n}\right\} \in \overline{\mathrm{GM}}$. Considering $k \in\left(N_{\xi}+1, N_{\xi+1}\right)$, we get

$$
\begin{aligned}
\sum_{l=k}^{\infty}\left|\Delta a_{l}\right| & \leq \sum_{j=\xi}^{\infty} \sum_{l=N_{j}+1}^{N_{j+1}}\left|\Delta a_{l}\right| \leq C \sum_{j=\xi}^{\infty} \frac{N_{j}}{10^{j}} \leq C \sum_{j=\xi+1}^{\infty} \frac{N_{j}}{10^{j}} \\
& \leq C \sum_{j=\xi+1}^{\infty} \frac{1}{10^{j}} \sum_{l=N_{j}+1}^{N_{j}+\left[N_{j} \exp \left(N_{j}\right)\right]} \frac{1}{l} \leq C \sum_{j=\xi+1}^{\infty} \sum_{l=N_{j}+1}^{N_{j+1}} \frac{\left|a_{l}\right|}{l} \leq C \sum_{l=k}^{\infty} \frac{\left|a_{l}\right|}{l} .
\end{aligned}
$$

Acknowledgements. This research was supported by MTM 2008-05561 -C02-02/MTM, RFFI 08-01-00302, and NSH-2787.2008.1. 


\section{References}

[AW] R. Askey and S. Wainger, Integrability theorems for Fourier series, Duke Math. J. 33 (1966), 223-228.

[Bab] K. I. Babenko, On conjugate functions, Dokl. Akad. Nauk SSSR 62 (1948). 157-160 (in Russian).

[Ba] N. K. Bary, A Treatise on Trigonometric Series, Vols. I, II, Pergamon Press, 1964.

[Be] A. S. Belov, Order estimates for best approximations and moduli of continuity of the sum of a trigonometric series with quasi-monotone coefficients, Math. Notes 51 (1992), 417-419; transl. from Mat. Zametki 51 (1992), no. 4, 132-134.

[Bo] R. P. Boas Jr., Integrability Theorems for Trigonometric Transforms, Springer, New York, 1967.

[CJ] T. W. Chaundy and A. E. Joliffe, The uniform convergence of a certain class of trigonometrical series, Proc. London Math. Soc. (2) 15 (1916), 214-216.

[DL] R. A. DeVore and G. G. Lorentz, Constructive Approximation, Springer, Berlin, 1993.

[DT 1 M. Dyachenko and S. Tikhonov, General monotone sequences and convergence of trigonometric series, in: Topics in Classical Analysis and Applications in Honor of Daniel Waterman, ed. by L. De Carli et al., World Sci., 2008, 88-101.

$\left[\mathrm{DT}_{2}\right]-,-$, Convergence of trigonometric series with general monotone coefficients, C. R. Math. Acad. Sci. Paris 345 (2007), 123-126.

$\left[\mathrm{He}_{1}\right]$ P. Heywood, On the integrability of functions defined by trigonometric series, Quart. J. Math. 5 (1954), 71-76.

[He $\mathrm{He}_{2} \quad-$, Integrability theorems for trigonometric series, ibid. 13 (1962), 172-180.

[HMW] R. Hunt, B. Muckenhoupt and R. Wheeden, Weighted norm inequalities for the conjugate function and Hilbert transform, Trans. Amer. Math. Soc. 176 (1973), $227-251$.

[LZ] R. J. Le and S. P. Zhou, A new condition for the uniform convergence of certain trigonometric series, Acta Math. Hungar. 108 (2005), 161-169.

[Le ${ }_{1}$ L. Leindler, Generalization of inequalities of Hardy and Littlewood, Acta Sci. Math. (Szeged) 31 (1970), 279-285.

$\left[\mathrm{Le}_{2}\right] \quad-$, On the uniform convergence and boundedness of a certain class of sine series, Anal. Math. 27 (2001), 279-285.

[Le $\left.{ }_{3}\right] \quad-$ Embedding results regarding strong approximation of sine series, Acta Sci. Math. (Szeged) 71 (2005), 91-103.

[Pi] H. R. Pitt, Theorems on Fourier series and power series, Duke Math. J. 3 (1937), 747-755.

[Po] M. K. Potapov and M. Berisha, Moduli of smoothness and the Fourier coefficients of periodic functions of one variable, Publ. Inst. Math. (Beograd) (N.S.) 26 (40) (1979), 215-228.

[St] E. Stein, Interpolation of linear operators, Trans. Amer. Math. Soc. 83 (1956), 482-492.

[Ti $\mathrm{Ti}_{1}$ S. Tikhonov, Trigonometric series with general monotone coefficients, J. Math. Anal. Appl. 326 (2007) 721-735.

$\left[\mathrm{Ti}_{2}\right] \quad$ - Trigonometric series of Nikol'skii classes, Acta Math. Hungar. 114 (2007), 61-78.

[ $\left.\mathrm{Ti}_{3}\right] \quad$ - Best approximation and moduli of smoothness: computation and equivalence theorems, J. Approx. Theory 153 (2008) 19-39. 
[YZZ] D. S. Yu, P. Zhou and S. P. Zhou, On $L^{p}$ integrability and convergence of trigonometric series, Studia Math. 182 (2007), 215-226.

[Zy] A. Zygmund, Trigonometric Series, Vols. I, II, 3rd ed., Cambridge Univ. Press, 2002.

Moscow State University

Vorob'evy Gory

Moscow 119992, Russia

E-mail: dyach@mail.ru
ICREA and Centre de Recerca Matemàtica

Apartat 50

08193 Bellaterra, Barcelona, Spain

E-mail: stikhonov@crm.cat

Received November 13, 2008

Revised version January 1, 2009 